\title{
Energy System Models for City Climate Mitigation Plans-Challenges and Recommendations
}

\author{
Burcu Unluturk and Anna Krook-Riekkola
}

\begin{abstract}
Many cities around the world have adopted climate neutrality targets, and, to reduce their greenhouse gas emissions, they need climate action plans. Energy system optimization models (ESOMs) can be used as tools to support their energy transitions. ESOMs have been in use at the national level for several years and also have recently been used at the city level. Even though several researchers have focused on how city ESOMs can be developed, the literature lacks a discussion of the challenges that are faced in data collection during model development. In this paper, we share the challenges encountered in the model development, as well as in the scenario development and recommend practical solutions for overcoming these challenges. The following three challenges were identified and discussed in the model development process: (a) data availability and quality; (b) communication; and (c) knowledge and background of civil servants and researchers. The main challenges in the scenario development were: (a) parameter selection and (b) complexity. It was found that explanation of the terminology used in ESOMs, presentation of the model structure and preliminary base-year results were crucial actions for overcoming challenges during model development. During the scenario development, collaboration between modelers and civil servants when reviewing parameter combinations and working with preliminary scenario results were decisive strategies for improving the civil servants' understanding of ESOMs. Complementarily, it was found that continuous communication between the researcher and the civil servant and good comprehension of the model on the municipality's side helped improve the usefulness of ESOMs in cities' energy transitions.
\end{abstract}

Keywords Climate mitigation plans $\cdot$ Energy transition $\cdot$ Energy system optimization models $\cdot$ Municipalities $\cdot$ Stakeholder engagement

B. Unluturk $(\bowtie) \cdot$ A. Krook-Riekkola

Energy Science Department, Luleå University of Technology, Luleå, Sweden

e-mail: burcuunluturk@gmail.com

A. Krook-Riekkola

e-mail: anna.krook-riekkola@ltu.se

(C) The Author(s) 2021

A. Bisello et al. (eds.), Smart and Sustainable Planning for Cities and Regions, Green Energy and Technology, https://doi.org/10.1007/978-3-030-57764-3_2 


\section{Introduction}

The global temperature rise is aimed to be limited to well below $2{ }^{\circ} \mathrm{C}$ above preindustrial levels with the Paris Agreement (Rogelj et al. 2016). Cities have a crucial role to play in achieving this aim since they are responsible for around $75 \%$ of the global greenhouse gas emissions (Edenhofer et al. 2014: 90). Many cities have committed to climate neutrality targets and have developed climate mitigation or adaptation plans, or both, to reduce emissions and prepare for climate change. At the time of writing, 9884 cities have signed the Covenant of Mayor agreement (Covenant of Mayors 2019). Under the agreement, cities develop climate mitigation and adaptation strategies, Sustainable Energy Climate Action Plans (SECAP) and commit to reducing $\mathrm{CO}_{2}$ emissions by at least $40 \%$ by the year 2030 (Covenant of Mayors 2019). In Europe, these strategies, plans and commitments are most common among cities with more than 500,000 inhabitants or among those that are signatories to the Covenant of Mayors, or both (Reckien et al. 2018).

In a comprehensive energy system optimization model (ESOM), various ways of achieving a transition to a climate-neutral energy system can be explored and assessed, taking local resources and other constraints into account. Therefore, ESOMs can be of benefit to climate mitigation plans. ESOMs have been used at the national level for a long time, e.g., UK TIMES (Daly and Fais 2014), TIMESPT (Simoes et al. 2008), TIMES-France (Maïzi and Assoumou 2014), TIMESSweden (Krook-Riekkola 2015), MESSAGE-Brazil (Nogueira de Oliveira 2016) and TEMBA (Taliotis et al. 2016). Recently, ESOMs are also increasingly being applied to cities, e.g., TIMES model for the city of Pesaro (Comodi, Cioccolanti and Gargiulo 2012), KomMod (Eggers and Stryi-Hipp 2013), TIMES-Oslo (Lind and Espegren 2017), INSMART (Simoes et al. 2019) and TIMES-city (Pardo-García et al. 2019). Model development and results from these models are available in the literature. Nevertheless, few literature contributions describe the process of developing or populating the model with city-specific data and information.

Models at national level are typically used to address national wide policies that can impact all kinds of energy use, while municipalities have a smaller room for maneuver. There is a need to develop a city-level model to support municipalities in decision making. Accordingly, city-level models, such as the generic TIMES-city model, should be structured to differentiate between aspects that municipalities can directly and indirectly impact (Krook-Riekkola et al. 2018). Furthermore, nationallevel models can be calibrated for base-year, based on national energy statistics that represent the energy supply and use of the country. On the other hand, the energy statistics on municipality level sometimes have another system boundary and do not have the desired granularity. The difference in system boundaries can be illustrated by the energy consumption for transportation, which is based on purchased fuels in the energy statistics. At the national level, this represents the fuel consumed by the citizens and companies, but it is not the case for the municipality level, i.e., since people can fuel their vehicle in another city. Lack of granularity of data in city-level energy statistics could be explained through an example: When there is only one 
company within a sub-sector, the energy use of this company is combined with a more aggregated sector, e.g., if there is one pulp and paper plant in the municipality, its energy use is only reported under industry sector, and not under pulp and paper sub-sector as in the national statistics. Therefore, it is not straightforward to populate a city-level model based on the official statistics. Another challenge is to identify the energy use by various users and activities and to split it between municipal and other use, thus breaking down the statistics into finer details. Consequently, a big challenge when developing city-level ESOMs is to make appropriate assumptions about the current energy system: The civil servants often have knowledge of the cities' energy systems but not the competence to identify the data needed to populate the model, while the energy system modelers have the energy system competence but not enough knowledge about the municipalities. Hence, the city-level modeling requires close communication between the cities and modelers.

In the SureCity project, a generic city-level ESOM (TIMES-city) was developed to support cities with reaching the climate targets of Covenant of Mayor (PardoGarcía et al. 2019). The TIMES-city model was developed considering cities' needs and their energy systems and engaging in continuous communication and collaboration with the municipalities during model development. The modeling process started with workshops in which civil servants and researchers came together and discussed the cities' needs, how the model could support their needs and which result indicators could be useful. These workshops were followed by model development and continuous communication, where researchers were responsible for developing the model and cities were responsible for providing the required information needed to populate the model.

The aim of our paper is to share the challenges that were encountered during data collection for model and scenario development and to recommend practical solutions for overcoming these challenges.

\section{Methodology}

\subsection{Energy System Optimization Models}

In the study, energy system optimization modeling based on The Integrated MARKAL-EFOM System (TIMES) modeling framework was used to describe existing and future energy systems at the city level. TIMES models are technologyrich and can be used for medium- to long-term scenario analysis and policy support (Loulou et al. 2016). They generally include all the steps from resource extraction, transformation, transport, distribution and energy conversion to supply of the cities' energy services (Loulou et al. 2016). In the case of TIMES-city, the researchers have developed the model based on city-specific information from the civil servants of each municipality. 


\subsection{The Generic TIMES-City Model}

In the generic TIMES-city model, nine sectors for service demands and their energy supply were included. These are energy supply, electricity generation, residential buildings, private commercial buildings, municipality managed buildings, waste, water and waste-water, public lighting, transport and industry (Krook-Riekkola et al. 2018). In the model, the urban energy system is defined as a Reference Energy System (RES). It is a schematic representation of technologies and energy commodities needed to meet the demand for energy-intensive goods and services (Krook-Riekkola et al. 2018). The calibration of the model was achieved with the city's existing technologies and infrastructure and energy use, whereas the database of the future technologies and fuel options are kept independent from the city. For the model, city administrative borders defined the boundaries for the determination of an activity's location, as inside or outside the city. Moreover, the energy, material and people flow going into and out of the city were included to represent the whole energy footprint of the city. Temporal resolution in the model makes it possible to see the variations between the years, and within a year and this leads to long-term and yearly assessments as mentioned in the Outline of the City-level Modeling framework report (Krook-Riekkola et al. 2018). Additionally, emission factors for greenhouse gases (GHGs) and air pollutants were included in the model which can be counted as another feature of the model to assess the environmental impact of the city.

\subsection{Scenario Generator}

The scenario generator is the part of the model where the municipality can define the scenarios they would like to assess. It is possible to change the following parameters to assess different pathways for the future: demand increase or decrease that is related to population and GDP, prices of fuels, electricity and district heating, $\mathrm{CO}_{2}$ mitigation levels (i.e., the target that a city sets to decrease its emissions rates from the calibration year of the model), availability of transport options in the future that the municipality can effect (e.g., public transport, car-pooling), building insulation and efficiency. In addition, it is possible to define scenarios with targets for the share of renewable energy sources and/or to only allow certain low-carbon technologies.

\subsection{Identification of Modeling Needs}

Comprehensive energy system models require a substantial amount of data. This data is not always straightforward to find, especially not on the city level. For this reason, we identified three different ambition levels in relation to data collection and modeling — rough, adequate and "perfect." The perfect model obviously does not 
exist and is not described further within this paper but was defined as a benchmark for the other two. The rough and the adequate models are further defined in this section.

A rough city-level ESOM is a model that does not have detailed city-specific data. We identified that the following information are needed in order to develop a rough model:

- Energy balance calibration based on aggregated city statistics, disaggregated based on expert knowledge.

- Aggregated demand per energy-intensive services, e.g., space heating.

- Demand projections based on growth index.

- Techno-economic-environmental characteristics of key technology options.

- Country policies and energy prices.

- Scenarios with varying climate targets.

An adequate city-level ESOM is a model populated with detailed city-level statistics. We identified the following information that is needed to develop the model:

- Scenarios with varying climate targets.

- Energy balance based on measured energy flows at the city level.

- Splitting demands into categories, e.g., space heating split into different building types.

- Demand projections based on city-specific drivers, e.g., population growth projection.

- Technology constraints.

- City-specific policy options.

- Thoroughly defined scenarios, based on cities' conditions, by civil servants with good understanding of how the scenario assumptions affect the model.

\section{Challenges of Using ESOMs at City Level}

From our experience working with cities with energy systems models, we identified particular challenges both when developing the model, i.e., in meeting the modeling requirements, and when developing scenarios.

\subsection{Identifying Challenges in Meeting Model Requirements}

We have realized some patterns that were challenging when developing the model. Sometimes, it concerned the data collection, and sometimes it was about the communication with the stakeholders (municipality officials). We categorized the challenges in meeting the modeling requirements as the following: data availability and quality, communication, knowledge and background. 
Data availability and quality: The existing data at the city level are mostly financial, while it is energy data that is needed for the model. For example, municipalities know how much they paid for fuel in a year for city-owned vehicles, but they do not necessarily know how much fuel they consumed. In another example, total electricity consumption in buildings was known, but the purchase distribution per building type was only documented in the economic system. Thus, the available unit of data is not in energy units. Data quality is crucial for base-year calibration, and good data quality is challenging to attain when demand and supply data do not match. This is especially the case when converting financial data to energy units. In the cases when collection is required by law and regulations, data is available. It has been straightforward to collect information on energy-related entities (e.g., district heating plants), even if the companies are privately owned, when their profession is energy. On the other hand, data on non-energy-related entities (e.g., schools), was more difficult to find. Final energy consumption can be directly measured in municipality-owned entities, but it is often not done. In these cases, when only aggregated energy statistics were available, these needed to be disaggregated in order to calibrate the model for the base-year.

Communication: It is crucial that cities understand their energy systems, the model and how they can benefit from it in order for these models to become useful. However, conveying this information is a complex task, since municipalities usually lack energy systems expertise and modelers generally do not have municipal insights. Thus, clear and continuous communication is required between the researcher and the city officials. Moreover, several models and data files are shared during model development. Therefore, finding ways to communicate the model and to share the files are important to have a smooth model development process.

Another challenge is the terminology used in the model and by the researcher, which can make it difficult for the civil servants to understand which data to collect unless there is a common language to communicate about the model. Misunderstanding the required data slows down the data collection and base-year calibration processes. For example, in energy system models, the difference between the final energy demand and the final energy consumption is important, but a civil servant who has not been working on ESOMs might not pay attention to this detail. Therefore, when the data required is not understood, the data collection process takes longer. Communication is crucial, and there is a need, in the beginning, to define how to communicate the project. Multiple ways of communication are needed for different purposes, e.g., file sharing, model explanation, clarification of required data, etc.

Knowledge and background: Civil servants have different backgrounds that may not be related to the field of energy. Even in the case when they have an energy-related background, our experience was that they rarely have energy system modeling expertise. Therefore, one of the main challenges to the collection of adequate information was that the civil servants providing the information had difficulties interpreting the energy system concepts because they lacked the relevant terminology. This sometimes meant that we retrieved the wrong kind of data and sometimes no data at all. This slowed down the process of data collection significantly. 


\subsection{Identifying Challenges in Working with Scenario Development}

In ESOMs, scenarios let us explore future pathways for, e.g., meeting specific climate targets under different conditions. Scenarios typically include coherent assumptions about the main drivers of the energy system and available energy resources, policy options and technologies, which should be internally consistent (Loulou et al. 2016: 10). In the scenario generator, the cities can define scenarios by combining assumptions about the various parameters. However, the scenario generator cannot serve its purpose if the parameters are not clearly understood by the civil servants. We wanted the civil servants to define their scenarios, but many times we saw that they had difficulty understanding how to select parameter combinations. The reason was due to the complexity of the model (capturing the comprehensive energy system, including both energy supply and demand sectors) and that they often wanted to see the impact from using a certain technology and/or energy source (while technologies and energy commodities are chosen by the model in this kind of scenario analysis). Consequently, the challenges are both in the parameter selection and in the complexity topics, and we elaborate them subsequently.

Parameter selection: The scenario generator includes several assumptions like change in demand, $\mathrm{CO}_{2}$-mitigation levels, policy options for the transportation sector, energy savings in the building sector, fuel prices, targets for renewable technology implementations and so on. These assumptions can be divided into two sub-groups: externalities that may impact city energy systems and assumptions that cities can directly impact. Examples of external parameters are: population evolution (impacting the living area, thus impacting the future demand for space heating and hot water), GDP (impacting purchase of goods, therefore impacting the demand on freight transportation) and fuel prices (a key assumption in the model). Examples of scenario parameters that the city can impact and may vary are: targets (e.g., renewable energy target for the purchased electricity; emission reduction target in transportation sector within city borders); and infrastructure (e.g., expansion of cycling infrastructure may impact the share of people who choose cycling instead of driving the car). Among all the various assumptions, selecting consistent parameters to define a scenario and collecting relevant data is challenging because it requires knowledge of national and local targets and conditions.

Complexity: How the parameters defined in the scenario generator may affect the overall energy system is not clear when the civil servants does not have experience of using ESOMs, which might lead to misunderstanding of the assumptions and the scenarios. Understanding the energy system and how various inputs affect the system is not easy, and it is not straightforward to explain. Interactions between the multiple parts of the system produce a complexity that is difficult to understand if you are not an expert. 


\section{Recommendations}

In response to the challenges identified in this paper, we share some recommendations based on our experience of using ESOM to support cities on their transition to lowcarbon energy systems. We categorize the recommendations in the same order as the challenges, data availability and quality, communication, knowledge and background for modeling requirements and the scenario assessment.

\subsection{Recommendations for the Challenges in Meeting Model Requirements}

Data availability and quality: Firstly, the data collection time should not be underestimated and should be considered in the project timeline because it might not be available in the desired unit or not available at all. In this case, a combined top-down and bottom-up approach is needed to retrieve the required data. For instance, total electricity consumption in buildings was known but purchase distribution per building type was only documented in the economic system. Thus, these two were used to obtain the required data. Similarly, in the transport sector, national average values were used in combination with information about fuel purchased for the population of the model.

Another approach could be to develop the model according to the available data that is not the most desired solution because the level of detail or desired scope might not be modeled. The most beneficial solution for the city and the modeler is having statistical documentation to ease the modeling load and to facilitate the civil servants to have correct information. To get better decision tools for working with city energy transitions, municipalities need to focus on statistical documentation of their final energy consumption per energy commodity and site, if possible, on hourly or monthly bases. This quite easy measure will significantly improve the quality of the analysis; hence, this is where the cities should prioritize putting their time.

Communication: To overcome the challenges, we identified various ways of communication in different parts of the process and for different needs. For file sharing, cloud services provide the most efficient solution according to our experience because the latest version of the file is available to every party at the same time. File sharing with e-mails is another option, which we recommend avoiding, as there is the possibility of using older file versions instead of current ones in model runs, thereby leading to attempts to re-correct problems that had already been encountered and overcome. For model explanation, we found online presentations by the researcher in combination with a report are useful for describing the model structure and explain the required data in detail. These were followed up with a question-and-answer session to support the understating from the city's side. For the model population, face-to-face meetings (physical or online) will speed up the 
process and are highly recommended based on both our own experience and feedback from the municipalities. A reason for holding face-to-face meeting, instead of using a chat forum or having a "voice-only-meeting" (a phone call or a Web-based meeting without camera), is to make it possible for the modeler to see the reaction of the civil servants to better apprehend if they have understood which data to collect and thereby avoid gathering the wrong information.

Knowledge and background: Civil servants can have different backgrounds and might not have an understanding of energy systems. We worked with several solutions to overcome this challenge. Initially, we tried collaborative-interactive meetings between researchers and civil servants for the base-year calibration. This can clarify many of the questions regarding the data population and can help eliminate the collection of wrong data. However, often the data to populate the model is not available during these initial meetings, so there is a need for several face-to-face meetings. Another approach that we used, and found more efficient, was showing preliminary results to the cities for the base-year of the model, and revealing the underlying preliminary data that had been gathered from available statistics and initial discussions with the municipality. This helped civil servants to get a better understanding of how the data, that is supposed to be gathered by them, is used in the model. This also contributed to municipalities' understanding of how the energy system is defined in the model. Furthermore, online or pre-recorded presentations of the file structure used for modeling are crucial for the cities to understand what kind of information is needed and where it is used. In addition, we put effort into explaining the required data as well as the energy concepts in the model templates to overcome the confusion that is caused by having a different understanding of the terminology than that used by the model developers.

\subsection{Recommendations for Scenario-Assessment Challenges}

The keys to achieving the aims of modeling are assuring to assist the civil servants to provide input for the scenarios and making sure that they understand the scenario analysis. Recommendations to the challenges mentioned in Sect. 3.2 are described here.

Parameter selection: For the cities to understand the outcomes from the scenario analysis, they need to be a part of the process of developing scenarios to reach their targets. To achieve successful assessments of the different pathways of how the energy system can evolve over time, cross-communication between modeling experts and civil servants is needed, both when defining and when analyzing the scenarios. In the cases, when civil servants are not experienced working with drivers (which we found is frequently the case), it is useful if the researchers first propose a way to define parameter combinations to form scenarios, which thereafter are discussed and revised in cooperation between researcher and civil servants. With regards to the parameters that the city can directly impact, it should be the other way around: First, the civil servant should propose the decisions that have been thought upon, 
e.g., to reduce their climate impact and, second, these should be discussed with the modelers with respect to how these can be captured in the model. Keep in mind that the model will identify cost-efficient technology and fuel options under each of the defined scenarios, thus this should typically not be varied in the scenario generator. Parameters used in the scenario generator of the TIMES-city model are listed to illustrate what kind of assumptions that the cities can work with while defining scenarios (see Table 1). In the scenario generator, there are three parameter variation options, namely, "high," "medium" and "low." These simple options made scenario definition easier for the cities. If an assumption is not going to be included in a scenario, the option to exclude it is defined as "none."

Complexity: To choose relevant scenario parameters, the city decision makers should ideally know how different parameters impact different parts of the energy system. One way to achieve this is to provide them with the Reference Energy System diagram of their city. However, this was not enough. Instead, it was found that the most efficient and practical solution was to define a simple scenario with a few targets for a preliminary model run and to provide example results to cities. To support this, well-thought-out visualization (good examples, reader-friendly graphs) will make it easier for the city to understand how the parameters that the city can influence may affect their future energy system. This also helps the cities see that they can make

Table 1 Scenario parameters defined in the scenario generator of the generic TIMES-city model

\begin{tabular}{l|l}
\hline Scenario parameters & Parameter variation options (rate of change) \\
\hline Socio-economic evolution (GDP/capita) & High/Medium/Low/None \\
\hline Population evolution (number of inhabitants) & High/Medium/Low/None \\
\hline Inhabited residential buildings variation $\left(\mathrm{m}^{2}\right)$ & High/Medium/Low/None \\
\hline $\begin{array}{l}\text { Variation of the electricity consumption per capita } \\
\text { of electric appliances in residential sector } \\
\text { (kWh/capita) }\end{array}$ & High/Medium/Low/None \\
\hline $\begin{array}{l}\text { Evolution rate of municipality growth (starting } \\
\text { year } 2015=100)\end{array}$ & High/Medium/Low/None \\
\hline $\begin{array}{l}\text { Variation in useful energy demand for commercial } \\
\text { sector/industry sector/public-lighting sector }\end{array}$ & High/Medium/Low/None \\
\hline \begin{tabular}{l} 
CO 2 mitigation level \\
\hline $\begin{array}{l}\text { Transport policy options based on population } \\
\text { evolution }\end{array}$
\end{tabular} & High/Medium/Low mitigation/None \\
\hline $\begin{array}{l}\text { Energy efficiency in residential buildings } \\
\text { Local renewable power and heat production }\end{array}$ & High/Medium/Low insulation/None \\
\hline $\begin{array}{l}\text { Fuel market } \\
\begin{array}{l}\text { Target renewable energy sector and low-carbon } \\
\text { technologies }\end{array}\end{array}$ & High/Medium/Low/None \\
\hline $\begin{array}{l}\text { Variation of the investment cost of the technology } \\
\text { for the user (this includes subsidies and taxes at } \\
\text { technology level) }\end{array}$ & High/Medium/Low/None \\
\hline
\end{tabular}


a change, when they are doubtful of their ability to have an impact on the energy transition. The most crucial recommendation to the modeler is to assist cities during the scenario-definition stage because this will help to focus on varying the scenario parameters with main impact on the results.

\section{Conclusion}

Cities have been working on climate mitigation and ESOMs are becoming more and more common at the city-level for supporting cities in their energy transition. In this paper, challenges in energy system modeling of cities in the data collection processes for model and scenario developments are discussed. For model development, we found that it is important to: (a) explain the terminology used and data required in the model in face-to-face meetings; (b) explain the structure of the model files through presentations and reports; and (c) provide preliminary results from the base-year as they are critical for overcoming the challenges pertaining to data availability and quality, communication, as well as knowledge and background. Similarly, to overcome the challenge of parameter selection and complexity in the scenario development, we found that it was crucial to: (a) review scenario parameter combinations in collaboration with the cities; and (b) provide examples with preliminary model results to show how city targets affect their energy systems. All the solutions provided in this paper have a common theme, i.e., continuous communication between the civil servant and the researcher. This helps to overcome the misunderstandings of the terminology used in ESOMs and creates a platform to describe the model structure for the city, which helps to improve the energy systems understanding of the city representatives. A better systems understating can support comprehension of what is critical for achieving the transition to a climate-neutral energy system and thereby robust decision making.

The workflow of the modeling process and the recommendations given in the paper are summarized in Fig. 1 that covers the steps from model description to scenario runs. It should be kept in mind that this is a simplified sketch to describe the process conceptually and several steps might require reiteration. 


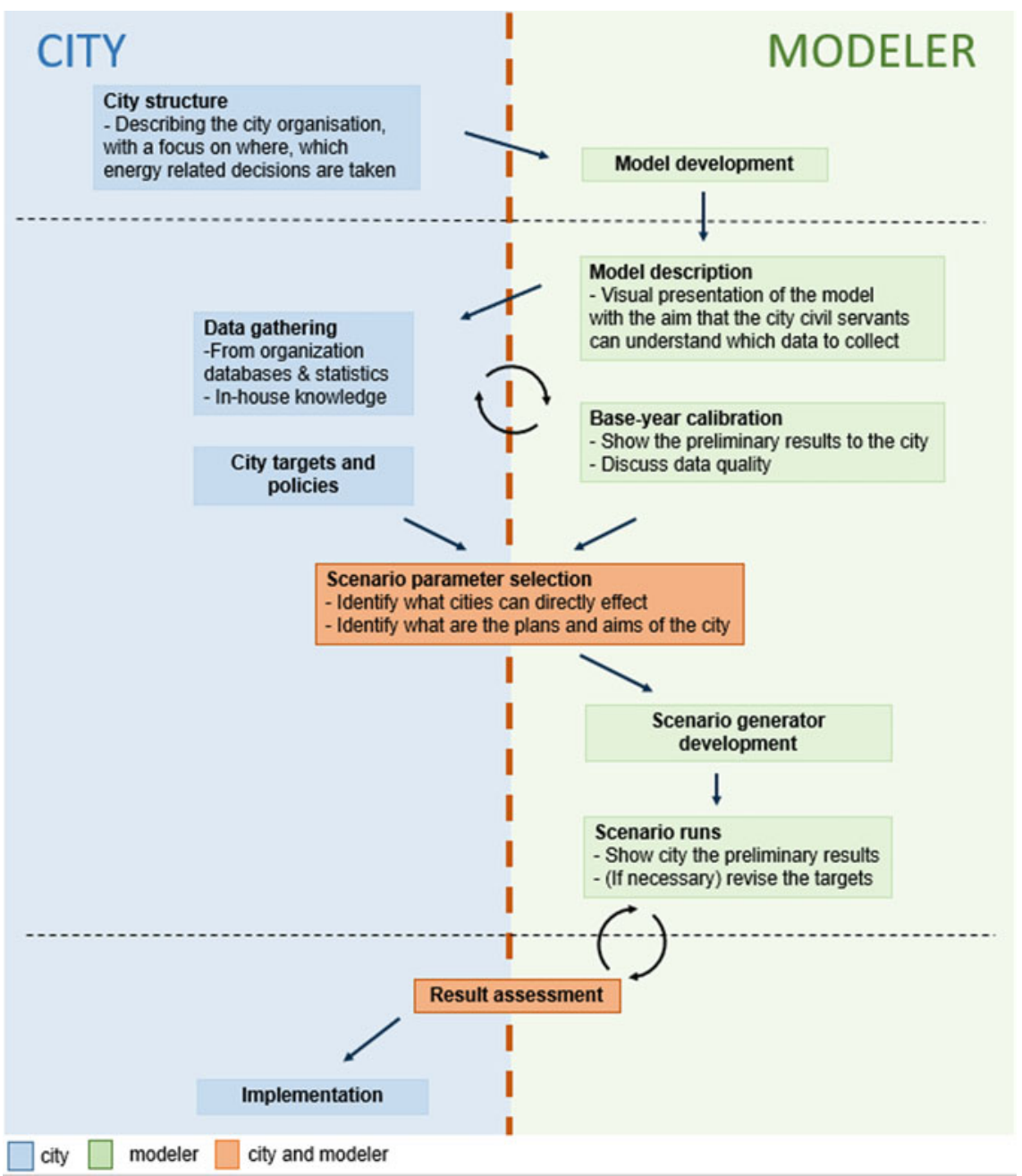

Fig. 1 Workflow on how civil servant and modelers can cooperate in modeling the city's energy system

Acknowledgements This study was carried out within the project "Swedish City's transition to a sustainable energy system-Can models support the decision-making process?", funded by the Swedish Energy Agency under the research program Människa, Energisystem och samhälle (Human, Energy and Society) (Energimyndigheten 2017). The authors acknowledge and thank the two anonymous reviewers for their constructive comments. Finally, we would like to thank our colleagues Jonas Forsberg and Carina Lundmark for the valuable discussions on the presented concept and Yawer Jafri for proofreading. 


\section{References}

Comodi G, Cioccolanti L, Gargiulo M (2012) Municipal scale scenario: analysis of an Italian seaside town with MarkAL-TIMES. Energy Policy 41:303-315

Covenant of Mayors (2019) Covenant initiative. https://www.covenantofmayors.eu/. Accessed 22 Nov 2019

Daly H, Fais B (2014) UK TIMES model overview. UCL Energy Institute. https://www.researchg ate.net/publication/267090898_The_UK_TIMES_Model_UKTM_Documentation. Accessed 10 Dec 2019

Eggers J-B, Stryi-Hipp G (2013) KomMod as a tool to support municipalities on their way to becoming smart energy cities. Sustainable buildings - construction products and technologies. In: Proceedings of the international sustainable building conference 2013, pp 580-591

Energimyndigheten (2017) Människa, Energisystem och samhälle. https://www.energimyndig heten.se/utlysningar/manniska-energisystem-och-samhalle/. Accessed 13 May 2020

Edenhofer O, Pichs-Madruga R,Sokona Y, Kadner S, Minx JC, Brunner S, Agrawala S, Baiocchi G, Bashmakov IA, Blanco G, Broome J, Bruckner T, Bustamante M, Clarke L, Conte Grand M, Creutzig F, Cruz-Núñez X, Dhakal S, Dubash NK, Eickemeier P, Farahani E, Fischedick M, Fleurbaey M, Gerlagh R, Gómez-Echeverri L, Gupta S, Harnisch J, Jiang K, Jotzo F, Kartha S, Klasen S, Kolstad C, Krey V, Kunreuther H, Lucon O, Masera O, Mulugetta Y, Norgaard RB, Patt A, Ravindranath NH, Riahi K, Roy J, Sagar A, Schaeffer R, Schlömer S, Seto KC, Seyboth K, Sims R, Smith P, Somanathan E, Stavins R, von Stechow C, Sterner T, Sugiyama T, Suh S, Ürge-Vorsatz D, Urama K, Venables A, Victor DG, Weber E, Zhou D, Zou J, Zwickel T (2014) Technical summary. Climate Change 2014: mitigation of climate change. Contribution of Working Group III to the Fifth Assessment Report of the Intergovernmental Panel on Climate Change [Edenhofer O, Pichs-Madruga R, Sokona Y, Farahani E, Kadner S, Seyboth K, Adler A, Baum I, Brunner S, Eickemeier P, Kriemann B, Savolainen J, Schlömer S, von Stechow C, Zwickel T, Minx JC (eds)]. Cambridge University Press, Cambridge, United Kingdom and New York, NY, USA

Krook-Riekkola A, Unluturk B, Forsberg J, Simoes S, Dias L, Garcia N (2018) Deliverable 3.1 Outline of the City-level modelling framework. https://surecityproject.eu/?q=file/52. Accessed 1 Dec 2019

Krook-Riekkola A (2015) National energy system modelling for supporting energy and climate policy decision-making: the case of Sweden. Chalmers University of Technology, Sweden

Lind A, Espegren K (2017) The use of energy system models for analysing the transition to lowcarbon cities - the case of Oslo. Energy Strategy Rev 15:44-56

Loulou R, Goldstein G, Kanudia A, Lettila A, Remme U (2016) Documentation for the TIMES Model Part I. https://iea-etsap.org/docs/Documentation_for_the_TIMES_Model-PartI_July-2016.pdf. Accessed 29 July 2019

Maïzi N, Assoumou E (2014) Future prospects for nuclear power in France. Appl Energy 136:849_ 859

Nogueira de Oliveira LP (2016) Temporal issuses in mitigation alternatives for the energy sector in Brazil. https://www.ppe.ufrj.br/images/publica\%C3\%A7\%C3\%B5es/doutorado/Larissa_Pupo_ Nogueira_de_Oliveira.pdf. Accessed 15 July 2019

Pardo-García N, Simoes S, Dias L, Sandgren A, Suna D, Krook-Riekkola A (2019) Sustainable and resource efficient cities platform-SureCity holistic simulation and optimization for smart cities. J Cleaner Prod 215:701-711

Reckien D, Salvia M, Heidrich O, Church J, Pietrapertosa F, De Gregorio-Hurtado S, D'Alonzo V, Foley A, Simoes S, Lorencová E, Orru H, Orru K, Wejs A, Flacke J, Olazabal M, Geneletti D, Feliu E, Vasilie S, Nador C, Krook-Riekkola A, Matosovic M, Fokaides PA, Ioannou BI, Flamos A, Spyridaki N, Balzan MV, Fülöp O, Paspaldzhiev I, Grafakos S, Dawson R (2018) How are cities planning to respond to climate change? Assessment of local climate plans from 885 cities in the EU-28. J Cleaner Prod 191:207-219 
Rogelj J, den Elzen M, Höhne N, Fransen T, Fekete H, Winkler H, Schaeffer R, Sha F, Riahi K, Meinshausen M (2016) Paris Agreement climate proposals need a boost to keep warming well below $2{ }^{\circ} \mathrm{C}$. Nature 534:631-639

Simoes SG, Dias L, Gouveia JP, Seixas J, De Miglio R, Chiodi A, Gargiulo M, Long G, Giannakidis G (2019) InSmart—a methodology for combining modelling with stakeholder input towards EU cities decarbonisation. J Cleaner Prod 231:428-445

Simoes S, Cleto J, Fortes P, Seixas J, Huppes G (2008) Cost of energy and environmental policy in Portuguese $\mathrm{CO}_{2}$ abatement—scenario analysis to 2020. Energy Policy 36:3598-3611

Taliotis C, Shivakumar A, Ramos E, Howells M, Mentis D, Sridharan V, Broad O, Mofor L (2016) An indicative analysis of investment opportunities in the African electricity supply sector-using TEMBA (The Electricity Model Base for Africa). Energy Sustain Dev 31:50-66

Open Access This chapter is licensed under the terms of the Creative Commons Attribution 4.0 International License (http://creativecommons.org/licenses/by/4.0/), which permits use, sharing, adaptation, distribution and reproduction in any medium or format, as long as you give appropriate credit to the original author(s) and the source, provide a link to the Creative Commons license and indicate if changes were made.

The images or other third party material in this chapter are included in the chapter's Creative Commons license, unless indicated otherwise in a credit line to the material. If material is not included in the chapter's Creative Commons license and your intended use is not permitted by statutory regulation or exceeds the permitted use, you will need to obtain permission directly from the copyright holder. 